\title{
Efficient Strategy For Processing Single Particle Data From a Cryo-EM Exposure Series
}

\author{
Robert A Grassucci ${ }^{*}$, William T. Baxter ${ }^{* *}$, Edward S. Barnard ${ }^{*, * * *}$ and Joachim Frank ${ }^{*, * *}$ \\ *Howard Hughes Medical Institute, Health Research, Inc. at the Wadsworth Center, Empire State \\ Plaza, Albany NY 12201-0509, USA. \\ ** Wadsworth Center, Empire State Plaza, Albany NY 12201-0509, USA. \\ ${ }^{* * *}$ Massachusetts Institute of Technology, Cambridge, MA 02139, USA
}

Processing data from a frozen hydrated biological sample is inherently difficult because of the low signal-to-noise ratio of the low electron dose images. This low dose is necessary in order to minimize the damage to the biological sample from exposure to the electron beam. A strategy has been developed to use a higher-signal image generated from the average of a series of low dose images for the majority of the processing sequence while still maintaining the ability to calculate a final reconstruction from the original low-dose data.

In order to study the effect of dose on biological samples, a series of four exposures were collected from the same area of a ribosome specimen at $300 \mathrm{kV}$ and 12 electrons $/ \AA^{2}$ for each exposure. The standard approach of extracting, aligning and reconstructing each of the four data sets separately was problematic because of the high noise level of the individual micrographs. Instead, the scanned micrographs were aligned to one another using high-contrast features so that the same particles could be selected from the averaged micrographs (Figure 1) using an automated particle picking procedure (Figure 2) [1]. Provided the number of particles is sufficient, the homogeneity of the sample, the E. coli ribosome-bound kirromycin-stalled aa-tRNA·EF-Tu·GDP ternary complex, should result in a high-resolution 3D reconstruction [2] (see Figure 3).

An investigation was done to determine how extensively the high-signal averages could be utilized without degrading the final reconstruction (Figure 4). Along with the increase in signal, using these averages also decreases the computation time when compared to processing each exposure separately.

The procedure used to generate a reconstruction consists of 4 major steps. In each of these steps one could potentially take advantage of the averaged images. Step 1 is particle picking, which we have determined works with the averaged micrographs as shown in Figure 2. Step 2 requires aligning and matching the experimental projections to the corresponding reference projections. This can be done with averaged particles, and the resulting output documents can be used to align and determine the projection angles for the single exposure particles. Step 3 uses the output of this step to generate a preliminary 3-D reconstruction. In step 4 the preliminary reconstruction is then used in place of the original reference to iteratively generate a series of refined volumes. A preliminary 3-D reconstruction from the averaged particles can be substituted and used as the starting reference to generate the refined volumes. The resolution of the various reconstructions are then compared to determine at what point use of the average data results in an inferior outcome [3].

References:

[1] B.K. Rath and J. Frank, J. Struct. Biol., 145 (2004) 84-90.

[2] M.Valle et al., Nature Structural Biology, 10 (2003) 899-906.

[3]This work was supported by HHMI and by NIH/NCRR grant P41 RR01219 (to JF). 


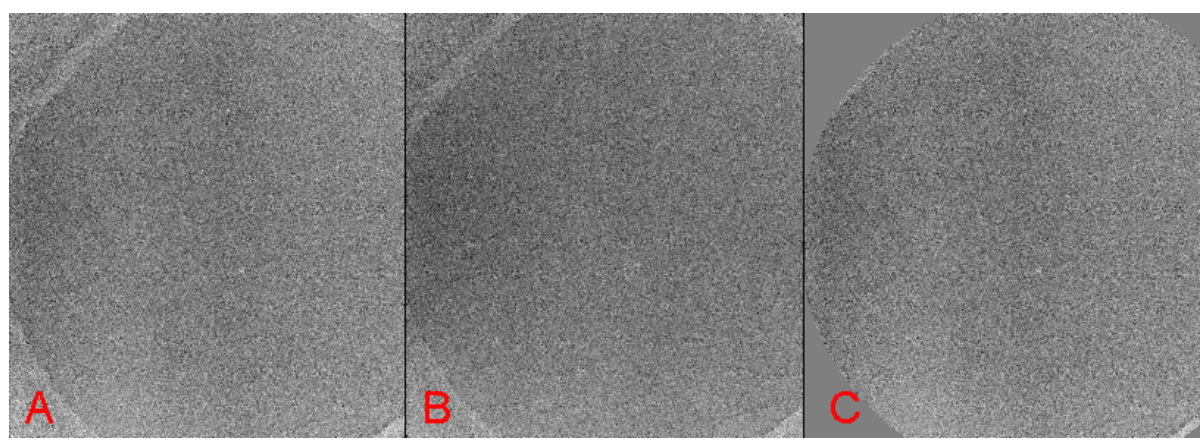

Figure 1. Aligned

Micrographs

A - Exposure 1

B - Exposure 2

C - Average of

Exposures 1-4 with

thick carbon foil

removed by masking

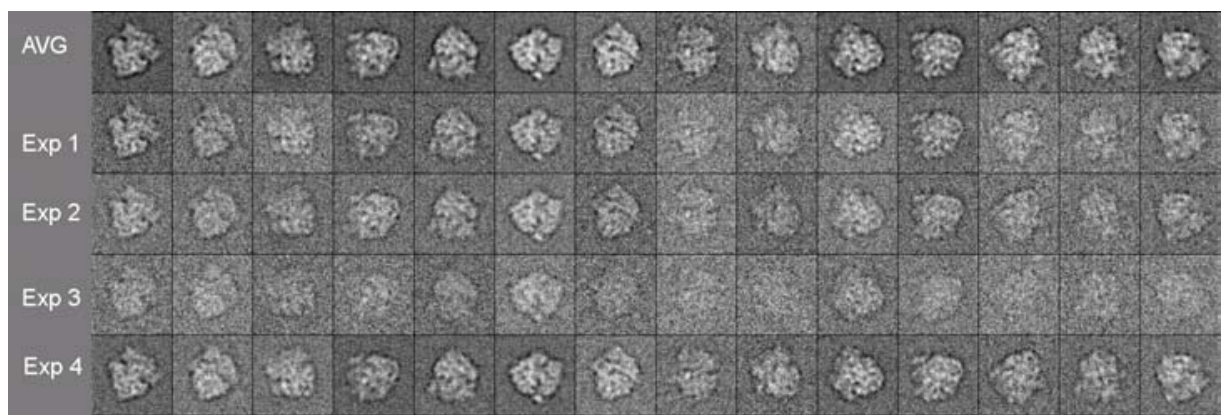

Figure 2. Montage of corresponding particles extracted from consecutive exposures using averaged micrograph. The top row shows an average of these individual projections.

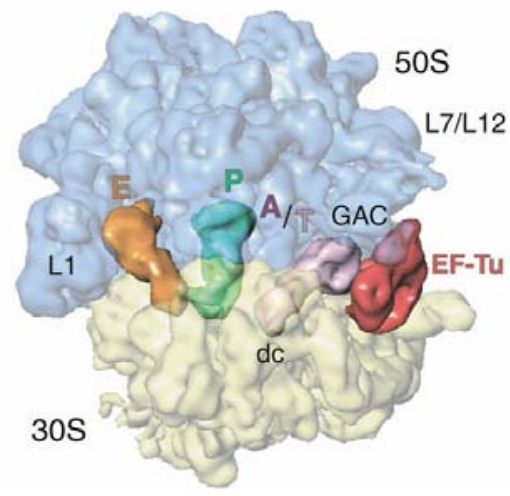

\section{Multiple Reconstructions}

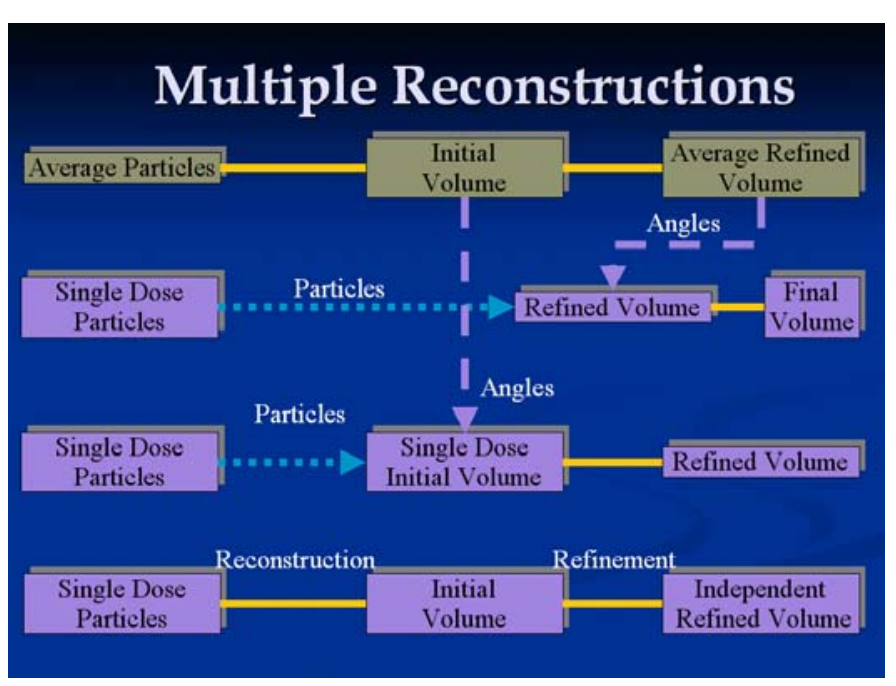

Figure 3. Surface rendering of 3D reconstruction from ribosome-bound kirromycin stalled ternary complex [2]

Figure 4. Flow diagram showing alternative processing paths Top Row - Average particles used throughout

Second Row - Angles from refined average used for refined single exposure volume

Third Row - Angles from initial averaged volume used for initial single-dose volume Last Row - Single-dose images reconstructed independently (control) 